\title{
Binding of Polycarboxylic Acids to Cationic Mixed Micelles: Effects of Polymer Counterion Binding and Polyion Charge Distribution
}

\author{
K. Yoshida, ${ }^{1}$ S. Sokhakian, and P. L. Dubin \\ Department of Chemistry, Indiana-Purdue University, Indianapolis, Indiana 46202
}

Received November 10, 1997; accepted May 1, 1998

\begin{abstract}
Mixed micelles of cetyltrimethylammonium chloride (CTAC) and $n$-dodecyl hexaoxyethylene glycol monoether $\left(C_{12} E_{8}\right)$ bind to polyanions when the mole fraction of the cationic surfactant exceeds a critical value $\left(Y_{\mathrm{c}}\right), Y_{\mathrm{c}}$ corresponds to a critical micelle surface charge density at which polyelectrolyte will bind to this colloidal particle. Turbidimetric titrations were used to determine $Y_{c}$ for such cationic-nonionic micelles in the presence of acrylic acid and acrylamido-2-methylpropane sulfonate homopolymers (PAA and PAMPS, respectively) and their copolymers with acrylamide, as function of $\mathrm{pH}$, ionic strength, and polyelectrolyte counterion. In $0.20 \mathrm{M} \mathrm{NaCl}, Y_{\mathrm{c}}$ for PAA is found to be remarkably insensitive to $\mathrm{pH}$, i.e., virtually independent of the apparent polymer charge density $\xi_{\text {app }}$. On the other hand, the expected inverse relationship between $Y_{c}$ and $\xi_{\text {app }}$ is observed either for PAA when $\mathrm{NaCl}$ is replaced by TMACI (tetramethylammonium chloride), or when $\xi_{\text {app }}$ is manipulated using acrylic acid/acrylamide copolymers at high $\mathrm{pH}$. The effective charge density of PAA is thus seen to be suppressed by specific sodium ion binding, indicating that the influence of salts on the interaction of polycarboxylic acids with colloidal particles may differ qualitatively from their effect on the analogous behavior of strong polyanions. Comparisons between homo- and copolymers of acrylic acid were carried out also to test the hypothesis that the "mobility" of charges on PAA at moderate $\mathrm{pH}$ (degree of ionization less than unity) could make this "annealed" polymer exhibit the behavior of a more highly charged one. The results, while consistent with this expectation, were obscured by the likely effect of copolymer sequence distributions. $\odot 1998$ A cademic Press
\end{abstract}

\section{INTRODUCTION}

Polyelectrolytes of moderate or high charge density associate with oppositely charged colloidal particles primarily via coulomb forces. It is, of course, difficult to exclude categorically the roles of hydrophobic interactions or hydrogen-bonding effects in these processes; however, the long-range nature of electrostatic forces, coupled with the difficulty of bringing multiple contiguous charged polymer residues close to the colloid surface, and the strong hydration of ionic groups on

\footnotetext{
${ }^{1}$ Permanent Address: Shiseido Corp., Yokohama, Japan.
}

both partners, all tend to diminish the influence of other, relatively short-range forces in such phenomena. The primacy of electrostatic forces is evident from (a) the diminution of complexation with increased ionic strength, $I$, and (b) the enhancement of the interaction with an increase in polyelectrolyte linear charge density, $\xi$, or colloid surface charge density, $\sigma$, and the concomitant absence of complexation when $\xi$, $\sigma$, or $I^{-1}$ are small.

The prevalence of electrostatic effects is clearly apparent from the properties of several polyelectrolyte-colloid systems in which complexation appears to exhibit phase transition-like behavior, i.e., critical conditions can be identified beyond which no complexes form, regardless of polyelectrolyte or colloid concentration. Such an adsorption-desorption transition was theoretically predicted for the adsorption of polyions on flat surfaces, first by Wiegel (1), and subsequently by Muthukumar (2) and Evers et al. (3); similar theoretical results were later obtained for strongly curved surfaces by Odijk (4) and Muthukumar (5), and also supported by simulations (6). In numerous studies with polyelectrolytes and oppositely charged micelles, we (7-10) have obtained a comparable empirical result, namely:

$$
\sigma_{\mathrm{c}} \cdot \xi \sim \kappa,
$$

where $\sigma_{\mathrm{c}}$ is the colloid critical surface charge density at the point of incipient complex formation, $\xi$ is the structural polymer linear charge density, and $\kappa$ is the Debye-Hückel parameter, proportional to $I^{1 / 2}$. This relationship also applies, at least at constant $\xi$, to the binding of proteins to polyelectrolytes (11) despite the fact that such binding occurs even when the protein net charge is of the same sign as the polymer's.

Complex formation between polyelectrolytes and various small colloids-micelles, proteins, dendrimers-has been studied in this group, using both polycations and polyanions, with a range of linear charge densities. Studies with micelles have been undertaken at surfactant concentrations typically two orders of magnitude above the critical micelle concentration (CMC), so that the relevant interaction is between polyelectrolyte and pre-existing micelle (not surfactant "monomer") and is thus to be differentiated from numerous studies of 
polymer-surfactant interactions carried out near or below the CMC. Indeed, polyelectrolyte-micelle complexation is more appropriately considered as a subset of polyelectrolyte-colloid interaction, and distinct from interactions of polymers with surfactants below the CMC. Included in the list of polyelectrolytes studied in combination with oppositely charged micelles are poly(acrylamidomethylpropanesulfonate) (PAMPS) and AMPS-acrylamide copolymers (10), poly(vinylsulfonate) (PVS) (12), poly(styrenesulfonate) (PSS) (13), poly(methacrylamidopropyltrimethylammonium chloride) (PMAPTAC) (14), sulfonated poly(vinylalcohol) (PVAS) (15), MAPTACacrylamide copolymers (16), and poly(dimethyldiallylammonium chloride) (PDADMAC) (17). All of these are strong polyelectrolytes, inasmuch as all ionic residues are fully dissociated, regardless of $\mathrm{pH}$. This property simplifies such studies, in that the colloid charge may be varied via the $\mathrm{pH}$, independently of the polymer charge. However, weak polyelectrolytes constitute a major class of water-soluble polymers, and the literature is rich in examples of their association with cahrged colloids. The earliest demonstration of "complex coacervation" involved two weak polyelectrolytes, gelatin and gum arabic (18), both naturally occurring polymers. Systems containing both weak polyelectrolytes and biopolymers have been studied in the context of protein separations: Morawetz (19), Glatz (20), and others (21) studied the phase separation of proteins with homo- and co-polymers of acrylic (AA) and methacrylic acid (MA). Related studies describe soluble complex formation between such polyacids and proteins (22), and binding of proteins to crosslinked acrylic acid copolymers (23). Fleer and coworkers (24) studied the adsorption of PAA onto cationic latexes. Studies of complex formation between polycarboxylate acids and micelles initially focused mainly on nonionic surfactants, where association is via hydrogen bonding, but more recent studies involve oppositely charged micelles, some of which have indicated qualitative differences between strong polyanions and polycarboxylic acids. For example, the binding of cationic surfactant micelles to PAA was found by Kwak to be much weaker than the binding to either PSS or dextran sulfate (25), clearly not a hydrophobic effect. Chu and Thomas (26) studied the binding of DeTAB to sodium polymethacrylate and found an increase in micelle aggregation number upon binding, opposite to the result obtained by Almgren et al. for DTAB and polystyrenesulfonate (27). Fundin et al. (28) found the aggregation number of CTAB bound to polyacrylic acid to be somewhat smaller than that of free micelles. Thalberg (29) measured the aggregation number of cationic micelles bound to sodium hyaluronate at $\mathrm{pH} 8$.

With regard to the adsorption of weak polyelectrolytes onto large colloids, several theoretical treatments have appeared (30). Some of the intriguing predictions have been: a maximum in the adsorbed amount as a function of $\mathrm{pH}$, and a very modest effect of ionic strength. The former effect was observed for a strongly cationic colloid (23), but not for the adsorption of PAA on a weakly cationic particle (31). Kinetic effects have also been examined, and the influence of chain length was studied for the adsorption of PAA onto weakly cationic Ba$\mathrm{TiO}_{3}(32)$.

Relatively few studies have provided a comparison of interactions with colloidal particles for strong vs weak polyelectrolytes. The primary issue in such comparisons has been of course the variable charge density of weak polyelectrolytes and their concomitant $\mathrm{pH}$-dependence, but it has also been pointed out that the $\mathrm{pK}$ of ionizable groups of weak polyions may be perturbed by the colloid surface potential. Fleer et al. (23) commented on the need to distinguish between the adsorption of strong and weak polyelectrolytes, and found that the effect of ionic strength on adsorption was far lower for weak polyelectrolytes. Shatayeva (33) discussed the relative binding of proteins to weak and strong cation exchange resins in terms of the acid strength of the network ionophores. Noting the larger binding energy reported for cationic surfactant micelles bound to PSS or dextran sulfate as compared to PAA (25), Li (34) suggested a specific difference between sulfonate vs carboxylate groups. However, aside from this observation, specific effects arising from differences among the ionogenic functional groups have hardly been considered.

In recent studies of complex formation between strong polycations and mixed nonionic/anionic micelles, we have noticed a prominent effect of the anionic surfactant head group. When surfactant sulfonate head groups are replaced by carboxylate head groups, complex formation with PDADMAC becomes more difficult to observe, even though $\sigma, \xi$, and $\kappa$ are unchanged (35). Some related studies were conducted with the same polycation but with a different negatively charged colloid: carboxyl-terminated dendrimers (36). For these solutes, we found that the relationship between the apparent surface charge density and the surface potential depends on the counterion (37): comparisons between $\mathrm{TMA}^{+}$and $\mathrm{Na}^{+}$provided strong evidence for the specific ion binding of $\mathrm{Na}^{+}$to dendrimer carboxylates. As will be made clear in the examples that follow, specific ion effects arise from factors other than the valency of the counterion, e.g., hydration or polarization.

Specific ion-binding effects for polycarboxylates are well known. Gregor pointed out many years ago that specific interactions do not occur between polycarboxylic acids and quaternary ammonium cations, or between polysulfonates and potassium ions, but "alkali metal cations of lower atomic weight engage in a specific binding reaction with anionic groups containing a strongly negative oxygen atom" (38). Such generalizations were confirmed by a variety of dilatometric and potentiometric studies for various polycarboxylic acids (39), supporting the general conclusions that the order of binding is $\mathrm{Li}^{+}>\mathrm{Na}^{+} \sim \mathrm{K}^{+}>>\mathrm{TMA}^{+}$. On the other hand, the theories for polyelectrolyte adsorption mentioned above do not take into account specific interactions between counterions and polyelectrolytes.

Counterion binding might be expected to perturb the relationship of Eq. [1] by diminishing the effective value of $\xi$. 
However, in studies in which $\xi$ was varied systematically by controlling the degree of sulfation, $S$, of polyvinylalcohol (14), the expected inverse dependence of $\sigma_{\text {crit }}$ on $S$ appeared. Certainly no evidence could be found to support any diminution of the effective polyelectrolyte charge density arising from counterion condensation at large $\xi$, inasmuch as such counterion condensation would lead to $\sigma_{\mathrm{c}}$ becoming independent of $S$ at large $S$. The theory of counterion condensation may not apply to polyelectrolytes in the potential domain arising from a nearby charged surface (40). Based on the foregoing discussion, however, effects of counterions might be more reasonably encountered for polycarboxylic acids. The primary goal of the present study was therefore to observe the influence of cationic counterions on the interaction between weak polyanions and positively charged micelles. In so doing, we compared polycarboxylic (weak) polyanions, and polysulfonate (strong) polyanions of equal formal charge density, in the presence of $\mathrm{Na}^{+}$and $\mathrm{TMA}^{+}$. Comparison between these two classes of polyanions has an additional dimension, in that they may be considered as "annealed" and "quenched" respectively, i.e., the charges on the weak polyacids can fluctuate due to the mobility of bound protons, whereas charges on the strong polyanions are essentially immutable. While counterion binding would be expected to diminish the effective charge on polycarboxylic acids, the fluctuations of charges can be viewed as a type of polarization effect, which may enhance interactions with oppositely charged micelles.

\section{EXPERIMENTAL}

Polyacrylic acid, PAA, (MW = 250,000, Aldrich) was neutralized with $\mathrm{NaOH}$, dialyzed and freeze-dried. Acrylic acid/ acrylamide, AA/AAm, (70/30) copolymer was purchased from General Science Co. (Tokyo), and dialyzed and freezedried before use. Poly(acrylamido-2-methylpropane sulfonate), PAMPS, and AMPS/AAm copolymers were prepared by polymerization of 2-(acrylamido)-2-methylpropanesulfonic acid with or without acrylamide as reported previously (41). Cetyltrimethylammonium chloride, CTAC, (Fluka) and Cetyltrimethylammonium bromide, CTAB, (Aldrich) were recrystallized twice from methanol. n-dodecyl hexaoxyethylene glycol monoether, $\mathrm{C}_{12} \mathrm{E}_{8}$, (Nikko Chemicals) was used as received. Tetramethylammonium chloride, TMAC, (Aldrich) was dried at $105^{\circ} \mathrm{C}$ overnight. Milli-Q water was used throughout this work.

Turbidimetric titrations were carried out at $420 \mathrm{~nm}$ using a Brinkman PC800 probe colorimeter equipped with a 1-cm path length fiber optics probe; all solutions were passed through $0.45 \mu \mathrm{m}$ Alltech filters before titrations. "Type I" turbidimetric titrations $(9,11)$, corresponding to the addition of ionic surfactant to polymer + nonionic surfactant micelle solutions, were performed at $22 \pm 2^{\circ} \mathrm{C}$ by adding $40 \mathrm{mM}$ cationic surfactant, either CTAB or CTAC, to a mixture of $0.50 \mathrm{~g} / \mathrm{L}$ polymer and $20 \mathrm{mM} \mathrm{C}_{12} \mathrm{E}_{8}$ at a constant ionic strength and $\mathrm{pH}$.

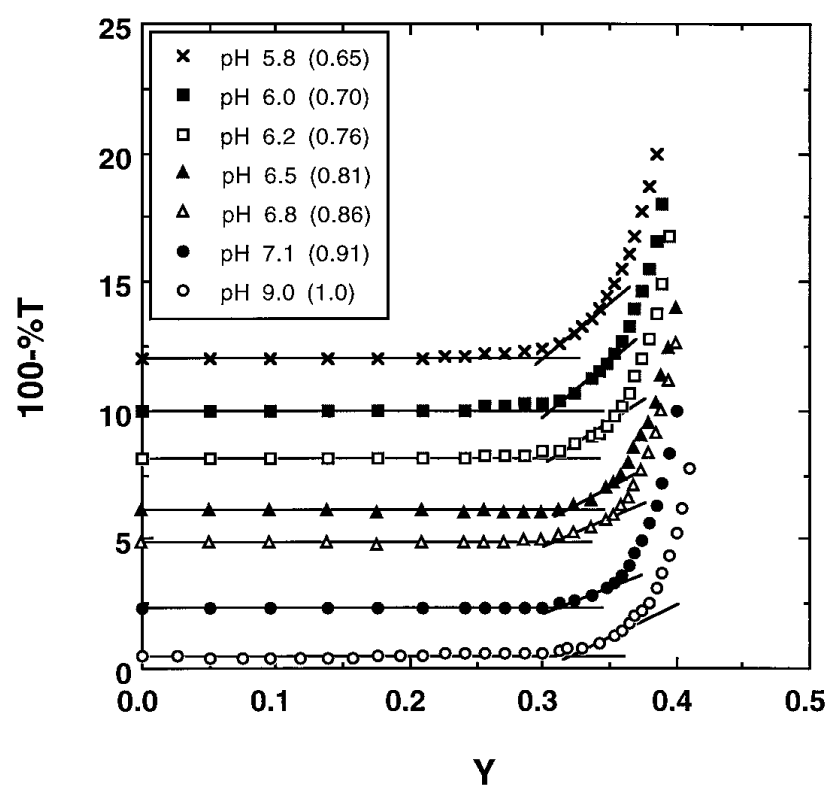

FIG. 1. "Type I" turbidimetric titrations (addition of $40 \mathrm{mM}$ CTAC to a mixture of $0.50 \mathrm{~g} / \mathrm{L}$ PAA and $20 \mathrm{mM} \mathrm{C}{ }_{12} \mathrm{E}_{8}$ at constant ionic strength) in various $\mathrm{pH}$ buffers at ionic strength $0.20 \mathrm{M}(0.19 \mathrm{M} \mathrm{NaCl}+$ buffer salt $) . Y$ is the mole fraction of CTAC in the mixed micelle. Turibidy (100-\% T) scale by addition of $2 \%$ for each titration curve (bottom to top) for clarity. $\mathrm{pH}(\alpha)$ values shown in insert.

We found no effect of the surfactant counterion $\left(\mathrm{Cl}^{-}, \mathrm{Br}^{-}\right)$on turbidimetric titrations, presumably because the final concentration of surfactant is small compared to that of supporting electrolyte. Ionic strength and $\mathrm{pH}$ were adjusted to the desired values with a mixture of simple salt (TMAC, $\mathrm{NaCl}$ ) and buffer salts $\left(\mathrm{pH}\right.$ 9.0, boric acid- $\mathrm{NaOH} ; \mathrm{pH}$ 6.8-7.1, $\mathrm{NaH}_{2} \mathrm{PO}_{4^{-}}$ $\mathrm{Na}_{2} \mathrm{HPO}_{4}$; pH 5.8-6.6, MES-NaOH) as well as $\mathrm{NaOH}$ and $\mathrm{HCl}$. The $\mathrm{pH}$ drift during titration, monitored by an Orion 811 $\mathrm{pH}$ meter equipped with a combination electrode, was within 0.05. All transmittance values were corrected by subtracting the turbidity of a polymer-free blank. The blank-corrected turbidity (100-T\%) was plotted vs $Y$, the mole fraction of ionic surfactant, defined as [cationic surfactant]/([cationic surfac$\operatorname{tant}]+[$ nonionic surfactant]).

\section{RESULTS AND DISCUSSION}

$\mathrm{PAA} / \mathrm{CTAC} / \mathrm{C}_{12} E_{8}$ in $\mathrm{NaCl}$. Since PAA is a weak polyelectrolyte whose titratable ionic groups have a $\mathrm{pH}$-dependent degree of ionization $(42,43), \alpha$, we can control the polymer linear charge density $(\xi)$ by fixing $\mathrm{pH}$. "Type I" turbidimetric titrations were carried out by adding $40 \mathrm{mM} \mathrm{CTAC}$ to $0.50 \mathrm{~g} / \mathrm{L}$ $\mathrm{NaPAA}+20 \mathrm{mM} \mathrm{C}_{12} \mathrm{E}_{8}$ in $I=0.20 \mathrm{M}(0.19 \mathrm{M} \mathrm{NaCl}+$ buffer salt) at constant $\mathrm{pH}$. In Fig. 1, the turbidity (reported as $100-\% \mathrm{~T}$ ) is plotted against $Y$, the mole fraction of CTAC in $\mathrm{CTAC} / \mathrm{C}_{12} \mathrm{E}_{8}$ mixed micelles. For pH $9.0(\alpha=1)$, the turbidity begins to increase at $Y=0.32$, corresponding to the onset of polymer-micelle interaction, i.e., $Y_{\mathrm{c}}$. That $Y_{\mathrm{c}}$, determined tur- 
bidimetrically, corresponds to the onset of polymer-micelle complexation has been verified by dynamic light scattering, electrophoretic light scattering, and fluorescence spectroscopy $(44,45)$. Surprisingly, $Y_{\mathrm{c}}$ values for lower $\mathrm{pH}(5.8-7.1)$ are the same as for $\mathrm{pH} 9.0$, even though the degrees of ionization at lower $\mathrm{pH}$ are substantially lower than 1.0. Since $Y_{\mathrm{c}}$ and $\alpha$ are expected to be proportional to $\sigma_{\mathrm{c}}$ and $\xi$, respectively, Eq. [1] leads to the prediction that $Y_{\mathrm{c}}$ should vary inversely with $\alpha$. The expected inverse dependence of $Y_{\mathrm{c}}$ on $\xi$ was indeed observed for the interaction of a strong polyanion with cationic mixed micelles: poly(AMPS-co-vinylpyrrolidone) copolymer (low $\xi$ ) showed larger $Y_{\mathrm{c}}$ than PAMPS homopolymer (high $\xi$ ) (9). The unexpected behavior of $Y_{\mathrm{c}}$ with $\alpha$ in present case is therefore of interest.

Three possible reasons may account for the anomalous behavior seen in Figure 1: First, according to Manning theory (46), polyelectrolyte counterion condensation in 1:1 electrolyte solution occurs if $\xi$ is greater than 1, and reduces $\xi_{\text {eff }}$ to unity. In the present case, $\xi_{\text {anal }}$ and $\xi_{\text {eff }}$ should diverge at $\alpha=0.35$, i.e., $\xi_{\text {eff }}=1$ at $\alpha \geq 0.35, \xi_{\text {eff }}=\alpha_{\text {anal }} \leq 1$ at $\alpha \leq 0.35$. In the above experiment, the lowest $\alpha(0.65$ at $\mathrm{pH} 5.8)$ is still above the critical linear charge density for counterion condensation. Unfortunately, we can not examine the range of lower $\alpha$ with the system PAA/CTAC/C ${ }_{12} \mathrm{E}_{8}$ since H-bonding of PAA with POE-type nonionic surfactant contributes to the polymer-micelle interaction at $\alpha<0.5$, introducing a complicating factor (47). Nevertheless, studies with strong polyelectrolytes appear to preclude an effect of counterion condensation on polymermicelle interaction. For example, although PAMPS homopolymer with poly(AMPS-co-vinylpyrrolidone) (PAMPS-VP) copolymer $(64 / 36)$ both have $\xi_{\text {anal }}>1$, the former binds more strongly to a cationic mixed micelle (lower $Y_{\mathrm{c}}$ ) at $I=0.1-$ 0.25 (9). Similarly, the critical micelle surface charge density for $81 \%$ sulfonated polystyrene sulfonate (PSS) is $\sim 10 \%$ larger than for $100 \%$ sulfonated PSS (48). In a related study, polyvinylalcohol sulfate (PVAS) with various degree of sulfonation $(S)$ were found to complex with cationic micelles of dimethyldodecylamineoxide at a critical degree of micelle protonation, $\beta_{\mathrm{c}}(35)$. Again, $\beta_{\mathrm{c}}$ varied inversely with $S$, even when $\xi_{\text {anal }}>1$. While it might be argued that partially sulfonated polystyrene could provide hydrophobic regions for interaction, this is surely not the case for the hydrophilic uncharged residues in PVAS or PAMPS-VP. This, and the fact that these systems all show the same linear dependence of $\sigma_{\mathrm{c}}$ on $I^{1 / 2}$, preclude the likelihood of a strong effect from nonelectrostatic interactions. Thus the systems previously studied indicate that the interaction between polyelectrolyte and micelle is predominantly electrostatic, but is not influenced by "Manning-type" counterion condensation onto the polyelectrolyte.

A second explanation for the unusual behavior of PAA/ $\mathrm{CTAC} / \mathrm{C}_{12} \mathrm{E}_{8}$ arises from consideration of specific counterion binding. As mentioned in the introduction, specific ion-binding for polycarboxylates is well known, and this counterion binding would also perturb the relationship of Eq. [1]. In particular,

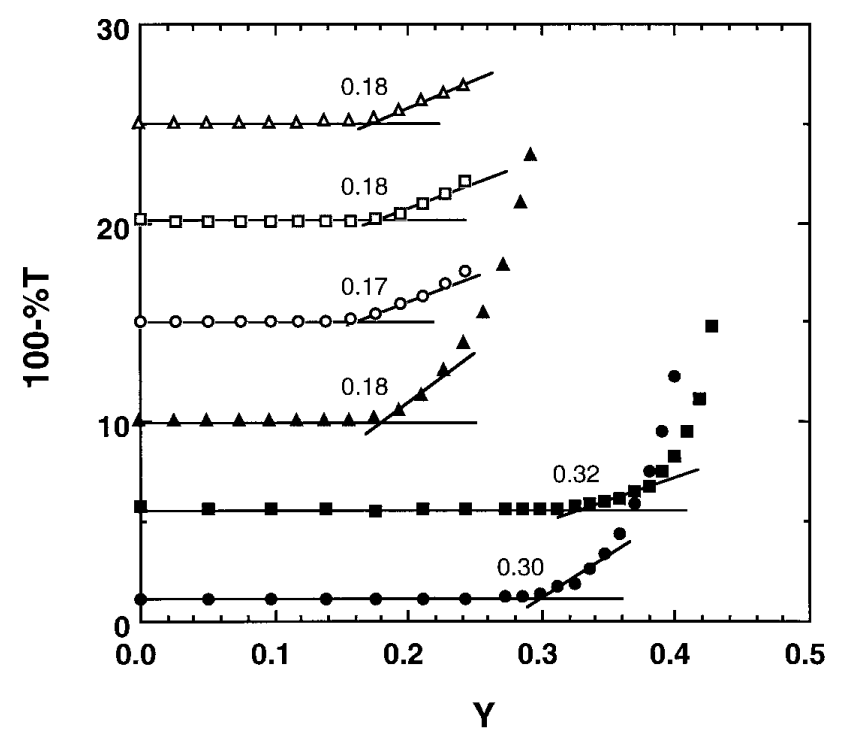

FIG. 2. Turbidimetric titrations for $0.50 \mathrm{~g} / \mathrm{L}$ NaPAMPS (open) or NaPAA (filled) $+20 \mathrm{mM} \mathrm{C}_{12} \mathrm{E}_{8}$ with $40 \mathrm{mM} \mathrm{CTAC}$ in $0.20 \mathrm{M} \mathrm{LiCl}(\bigcirc, \bullet) / \mathrm{NaCl}(\square$, $\mathbf{\square}) / \mathrm{TMAC}(\triangle, \mathbf{\Delta})$, all at $\mathrm{pH} 9.0$. Turbidity $(100-\% \mathrm{~T})$ scale adjusted as in Fig. 1.

small alkali metal ions bind to polyacrylic acid strongly (49, 50). This counterion binding could lead to a leveling of $\xi_{\text {eff }}$, and consequently $Y_{\mathrm{c}}$ would become insensitive to $\xi_{\mathrm{anal}}$. It is interesting to note that Kiefer et al. found that isotherms for the binding of (monomeric) tetradecyltrimethylammonium bromide to polyacrylic acid, in $0.01 \mathrm{M} \mathrm{NaBr}$, were essentially independent of the degree of neutralization of PAA, above $\alpha=$ 0.5 ; they also concluded that the effective charge density of PAA at large $\alpha$ is reduced by counterion binding (51).

A third phenomenon that could complicate the relationship between $\alpha$ and $\xi_{\text {eff }}$ arises from the mobility of the charge in PAA. Each monomer unit of PAA is identical, and the charge depends on the binding of $\mathrm{H}^{+}$, which is labile. Consequently, the local polymer linear charge density may not be simply proportional to the average degree of ionization. Indeed, $\xi_{\text {eff }}$ for $\alpha<1.0$ might be the same as that of fully dissociated PAA. This "charge annealing" effect is a form of polarization that can lead to an unexpectedly low value of $Y_{\mathrm{c}}$.

Effect of counterion type on strong and weak polyelectrolytes. Type $I$ turbidimetric titrations for $0.50 \mathrm{~g} / \mathrm{L} \mathrm{NaPAMPS}$ or NaPAA $+20 \mathrm{mM} \mathrm{C}_{12} \mathrm{E}_{8}$ with $40 \mathrm{mM} \mathrm{CTAC}$ were carried out in $0.20 \mathrm{M} \mathrm{LiCl}, \mathrm{NaCl}$ or TMAC at $\mathrm{pH} 9.0$, with the results shown in Fig. 2. $Y_{\mathrm{c}}$ for PAMPS was found to be $\cong 0.18$, independent of the counterion. The same value of $Y_{\mathrm{c}}$ was found for PAA in 0.20 M TMAC. Substantially larger values for $Y_{\mathrm{c}}$ were obtained for PAA in $0.20 \mathrm{M} \mathrm{NaCl}$ or $\mathrm{LiCl}$; this result suggests that $\xi_{\text {eff }}$ of PAA is significantly reduced by $\mathrm{Li}^{+}$and $\mathrm{Na}^{+}$binding. The stronger binding to PAA of $\mathrm{Li}^{+}$and $\mathrm{Na}^{+}$ relative to $\mathrm{TMA}^{+}$is consistent with the results of potentiometric studies $(34 \mathrm{c}, 45,46)$ for other polycarboxylic acids, whereas PAMPS, with bulky, low charge density sulfonate 


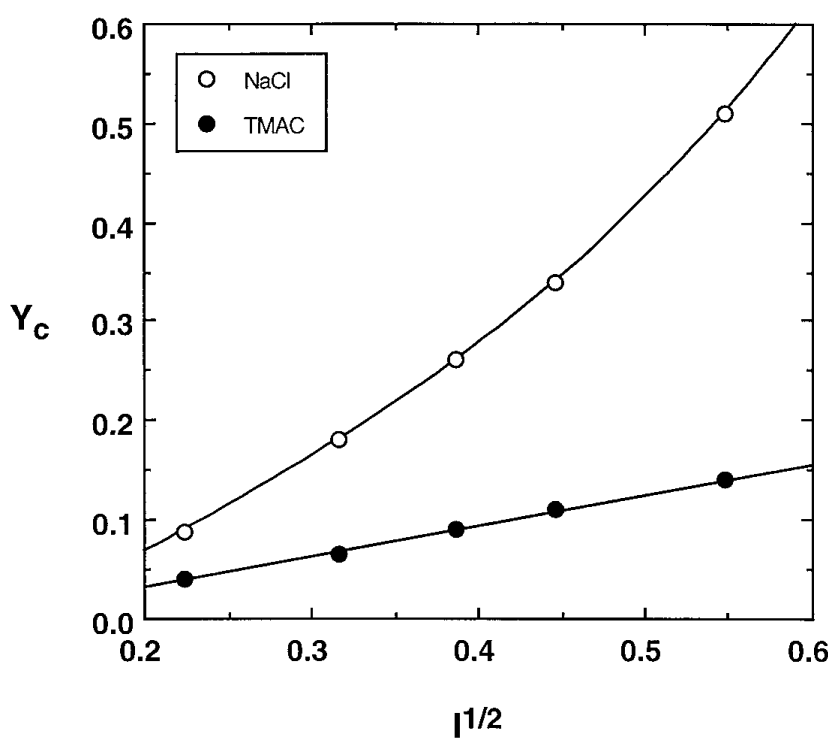

FIG. 3. Ionic strength dependence of $Y_{\mathrm{c}}$ for $\mathrm{PAA} / \mathrm{CTAB} / \mathrm{C}_{12} \mathrm{E}_{8}$ in $\mathrm{NaCl}$ and TMAC.

(large radius) groups, appears to be insensitive to this effect. The result of $\mathrm{Li}^{+}$and $\mathrm{Na}^{+}$binding to PAA is thus a "leveling" of $\xi_{\text {eff }}$ so that no effect of $\alpha$ on $Y_{\mathrm{c}}$ can be seen. Even though early potentiometric titration data suggested that $\mathrm{Li}^{+}$binds to PAA more storngly than $\mathrm{Na}^{+}(36 \mathrm{e}, 46)$, our results suggest that $\mathrm{Na}^{+}$reduces $\xi_{\text {eff }}$ more effectively than $\mathrm{Li}^{+}$. We note that the relative degrees of counterion binding result from both ion bulkiness and from hydration, for which the sequence $\left(\mathrm{Li}^{+}>\right.$ $\mathrm{Na}^{+}>\mathrm{TMA}^{+}$) (52) is opposite to the effect of ion size. The relative importance of these two effects for polycarboxylates at oppositely charged surfaces may differ from their contributions to counterion binding of the isolated polyion.

Effect of ionic strength. Turbidity titrations of $0.50 \mathrm{~g} / \mathrm{L}$ $\mathrm{NaPAA}+20 \mathrm{mM} \mathrm{C}_{12} \mathrm{E}_{8}$ at $\mathrm{pH} 9.0$ in $\mathrm{NaCl}$ and TMAC of varying ionic strengths were conducted using $40 \mathrm{mM} \mathrm{CTAB}$ as titrant. $Y_{\mathrm{c}}$ values, obtained as in Figs. 1 and 2, are plotted against $I^{1 / 2}$ in Fig. 3. In the presence of TMAC, we find the same linear dependence of $Y_{\mathrm{c}}$ on $I^{1 / 2}$ as revealed in previous studies on a number of polyelectrolyte-micelle systems $(9,11$, 53). This linearity suggests that the phenomenon is governed by Eq. [1], i.e., purely electrostatic. It also suggests that the only role played by $\mathrm{TMA}^{+}$is as a screening electrolyte, precluding its solubilization in the micelle (54). However, in $\mathrm{NaCl}, Y_{\mathrm{c}}$ is substantially larger than in TMAC, with the difference increasing with ionic strength. This result suggests that the degree of $\mathrm{Na}^{+}$binding increases with increasing $\mathrm{Na}^{+}$ concentrations, leading to reduction of $\xi_{\text {eff }}$ at higher ionic strength. The nonlinear relationship between critical colloid surface charge density and $I^{1 / 2}$ was also seen for the binding of a strong polycation (PDADMAC) to carboxyl-terminated dendrimer (55) in the presence of $\mathrm{NaCl}$.

The difference between $Y_{\mathrm{c}}$ in $\mathrm{NaCl}$ and in TMAC depends on the degree of $\mathrm{Na}^{+}$binding, and the concomitant reduction of $\alpha_{\text {eff }}$ by $\mathrm{Na}^{+}$. Since $\sigma_{\mathrm{c}}$ is proportional to $Y_{\mathrm{c}}$, and $\kappa$ to $I^{1 / 2}$, Eq. [1] becomes:

$$
Y_{\mathrm{c}} \sim \kappa \cdot \xi_{\mathrm{eff}}^{-1}
$$

Thus,

$$
\begin{gathered}
Y_{\mathrm{c}}^{\mathrm{TMA}} \sim \kappa \cdot \xi_{\mathrm{eff}, \mathrm{TMA}}^{-1}, \\
Y_{\mathrm{c}}^{\mathrm{Na}} \sim \kappa \cdot \xi_{\mathrm{eff}, \mathrm{Na}}^{-1},
\end{gathered}
$$

and, assuming the proportionality constant to be invariant,

$$
\frac{\xi_{\text {eff,Na }}}{\xi_{\text {eff,TMA }}}=\frac{Y_{\mathrm{c}}^{\mathrm{Na}}}{Y_{\mathrm{c}}^{\mathrm{TMA}}} .
$$

Values for $Y_{\mathrm{c}}$ in $\mathrm{NaCl}$ and TMAC at varying ionic strength and for $\xi_{\text {eff,NaCl }} / \xi_{\text {eff,TMAC }}$ are summarized in Table 1 . Since $Y_{\mathrm{c}}$ is identical for $\mathrm{Li}^{+}, \mathrm{Na}^{+}$, and $\mathrm{TMA}^{+}$in the case of PAMPS (Fig. 2 ), we must conclude that if $\xi_{\text {eff }}$-as manifested in the polyelectrolyte-micelle interaction-is reduced by counterion binding, this effect is independent of ion type; since an effect of polymer structural charge density is observed well above the "Manning" $\xi_{\text {crit }}$, the reasonable conclusion is that there is no ion-binding effect on $Y_{\mathrm{c}}$ for any of these cations with PAMPS. Figure 2 also shows identical values of $Y_{c}$ for PAA and PAMPS when the counterion is $\mathrm{TMA}^{+}$. If we then assume that $\mathrm{TMA}^{+}$is not bound to either PAMPS or PAA, then we conclude from comparison of those results to the behavior of the PAA/ $\mathrm{NaCl}$ system, that the effective charge of PAA is reduced by $50 \%$ in $0.050 \mathrm{M} \mathrm{NaCl}$ and by $70 \%$ in $0.30 \mathrm{M} \mathrm{NaCl}$.

Effect of polymer charge mobility. Since the charge density of PAA depends on the binding of $\mathrm{H}^{+}$, which is labile, the local polymer linear charge density may not be proportional to the average degree of ionization. The fluctuating local charge on PAA may be described as "annealed" in contrast to the fixed charge on a strong polyelectrolyte like PAMPS, which may be described as "quenched" (56). Since $\mathrm{Na}^{+}$binding makes it difficult to resolve the effects of counterion binding and charge annealing, we compared PAA at pH $6.6(\alpha=0.70)$ with PAA-co-AAm (70/30) at pH $9.0(\alpha=1.0)$ in TMAC. Both

TABLE 1

E ffect of Polymer Counterion Concentrations on $Y_{\mathrm{c}}$ for PAA and Effective Polymer Linear C harge Density Ratios

\begin{tabular}{clll}
\hline Salt $(\mathrm{M})$ & $Y_{\mathrm{c}}^{\mathrm{TMAC}}$ & $Y_{\mathrm{c}}^{\mathrm{NaCl}}$ & $\chi_{\mathrm{eff}}^{\mathrm{NaCl}} / \xi_{\mathrm{eff}}^{\mathrm{TMAC}}$ \\
\hline 0.050 & 0.041 & 0.089 & 0.46 \\
0.10 & 0.065 & 0.18 & 0.36 \\
0.15 & & 0.26 & \\
0.20 & & 0.34 & 0.27 \\
0.30 & 0.14 & 0.51 & 0 \\
\hline
\end{tabular}




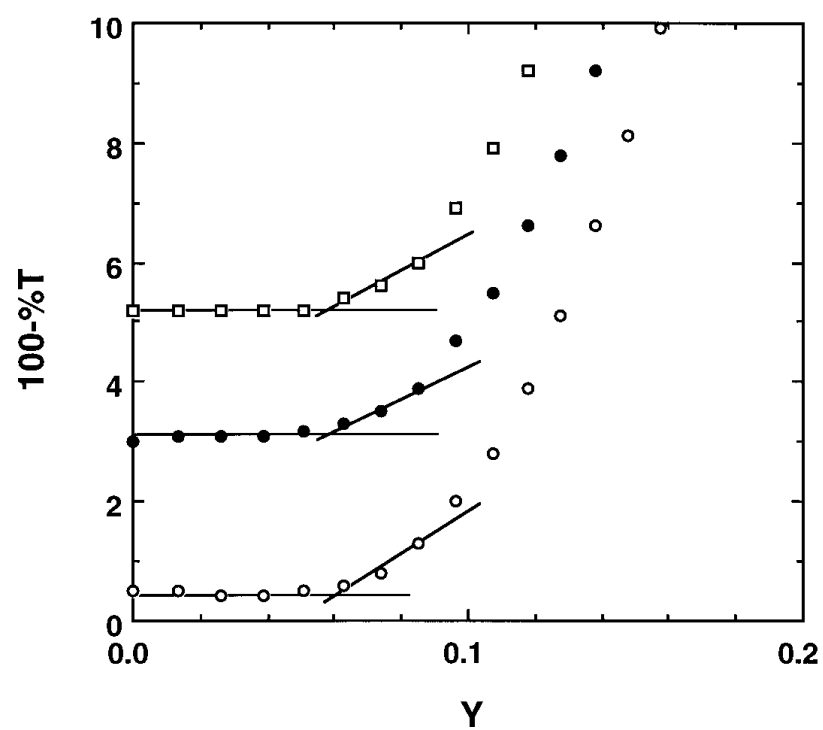

FIG. 4. Turbidimetric titrations for $0.50 \mathrm{~g} / \mathrm{L}$ NaPAA or NaPAA-coAAm $+20 \mathrm{mM} \mathrm{C}_{12} \mathrm{E}_{8}$ with $40 \mathrm{mM} \mathrm{CTAC}$ at ionic strength $0.10 \mathrm{M}(0.090 \mathrm{M}$ TMAC + buffer). $(\bigcirc)$ NaPAA at pH $9.0(\alpha=1.0)$; $(\bigcirc)$ NaPAA-co-AAm (70/30) at pH 9.0; $(\square)$ NaPAA at pH $6.6(\alpha=0.70)$. Turbidity (100-\% T) scale adjusted as in Fig. 1.

polymers have the same analytical charge density, but the former is annealed, the latter quenched.

Figure 4 shows type I turbidimetric titrations for NaPAA at $\mathrm{pH}$ 9.0, PAA-co-AAm (70/30) at $\mathrm{pH} 9.0$, and PAA at $\mathrm{pH} 6.6$ $(\alpha=0.70)$, all in the presence of $20 \mathrm{mM} \mathrm{C}_{12} \mathrm{E}_{8}$ and $I=0.10$ M (0.090 M TMAC + buffer salt), using $40 \mathrm{mM} \mathrm{CTAC} \mathrm{as}$ titrant. The second and third of these three polymers should have the same mean linear charge densities, while the first should be larger. Surprisingly, all three $Y_{\mathrm{c}}$ values were identical $\left(Y_{\mathrm{c}}=0.06\right)$, even though the values of $\xi_{\text {anal }}$ for PAA-coAAm at pH 9.0 and for PAA at pH 6.6 are both $30 \%$ smaller than that for PAA at $\mathrm{pH} 9.0$.

For both PAA and PAA-co-AAm at $\mathrm{pH}=9.0>>\mathrm{pK}_{\mathrm{a}}$, we can consider all carboxyl groups to be fully ionized, so that $\xi_{\text {eff }}$ $=\xi_{\text {anal }}$. However, in the case of PAA at $\mathrm{pH} 6.6, \xi_{\text {eff }}$ may not be the same as $\xi_{\text {anal }}$ because of polymer charge mobility. Therefore, the local $\xi$ at $\alpha<1.0$ might be the same as that of fully dissociated PAA. The situation under consideration is represented schematically in Fig. 5, in which $70 \%$ of the carboxyl groups are ionized ( $\alpha=0.70)$. PAA adjusts its charge distribution when it approaches an oppositely charged micelle, making it possible to bind like a highly charged polyion. The same $Y_{\mathrm{c}}$ for PAA at $\mathrm{pH} 9.0$ and 6.6 suggests that the polymer charge groups can move and tune $\xi_{\text {eff }}$ to maximize the binding ability. This polarization effect should be differentiated from a pK shift for the carboxylic acids induced by the proximity of the micelle, which apparently is rather small (28). However, this polarization mechanism would not apply to PAA-co-AAm $(70 / 30)$ at $\mathrm{pH}=9.0$, and thus leaves unexplained the identical values of $Y_{\mathrm{c}}$ seen for this copolymer and PAA at $\mathrm{pH}$ 9.0.
Copolymer compositional and sequential distribution. The identical values of $Y_{\mathrm{c}}$ obtained for NaPAA and PAA-co-AAm (70/30) at $\mathrm{pH} 9.0$ cannot be explained by charge migration, but could arise from the nature of the composition of the copolymer. Since copolymer composition is based on the instantaneous mole ratio of monomers during polymerization, copolymers may have (1) compositional and (2) sequential distributions. Those distributions can lower $Y_{\mathrm{c}}$ because (1) a copolymer enriched in ionic comonomer and therefore of higher $\xi$ binds to micelles earlier, and (2) partially continuous ionic group sequences (small blocks) also can bind to micelles at low $Y$. In order to examine this issue, without complications arising from counterion binding, H-bonding, or charge mobility, we carried out turbidity titrations using a series of AMPS-co-AAm polymers.

The results of turbidity titrations for PAMPS-co-AAm of varying AMPS contents are shown in Fig. 6. All copolymers indeed show larger $Y_{\mathrm{c}}$ than that of homopolymer, indicating lower $\xi_{\text {eff. }}$. However, if $\xi_{\text {eff }} \sim \%$ AMPS, and $\sigma_{\mathrm{c}} \sim Y_{\mathrm{c}}$, then we should find $Y_{\mathrm{c}} \sim(\% \text { AMPS })^{-1}$. The dependence of $Y_{\mathrm{c}}$ on $\%$ AMPS is shown in Fig. 7, where the dashed line is $Y_{\mathrm{c}}=$ constant (\% AMPS) ${ }^{-1}$, with the constant obtained as 18 based on $Y_{\mathrm{c}}$ found for $100 \%$ AMPS. $Y_{\mathrm{c}}$ is seen to deviate from this
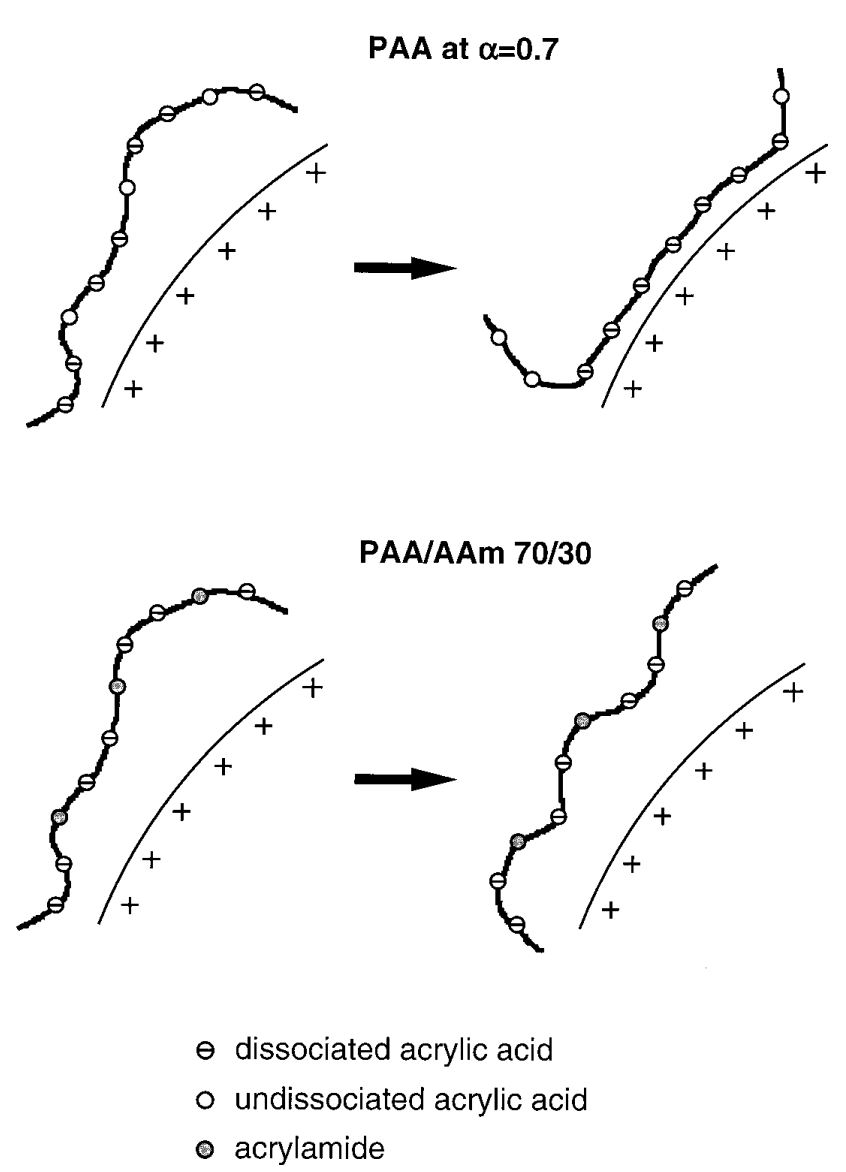

FIG. 5. Schematic depiction of the behavior of quenched and annealed polymer charges on binding to micelles. 
line, especially at low \% AMPS, indicating $\xi_{\text {eff }}>\xi_{\text {anal }}$. In particular, $Y_{\mathrm{c}}$ is unexpectedly insensitive to AMPS content and varies somewhat erratically with \% AMPS. Similar behavior has been observed for these same copolymers and DMDAO micelles: here $\mathrm{pH}_{\mathrm{c}}$ is found to increase, as expected, with \% AMPS, but the dependence is weak (57). We attribute this "leveling effect" to compositional and/or sequential distribution in the polymer chain. The monomer reactivity ratios of AMPS(rl)/AAm(r2) = 1.00/0.92 (58) are in fact those of an ideal random copolymer in which the tendency to form "runs"

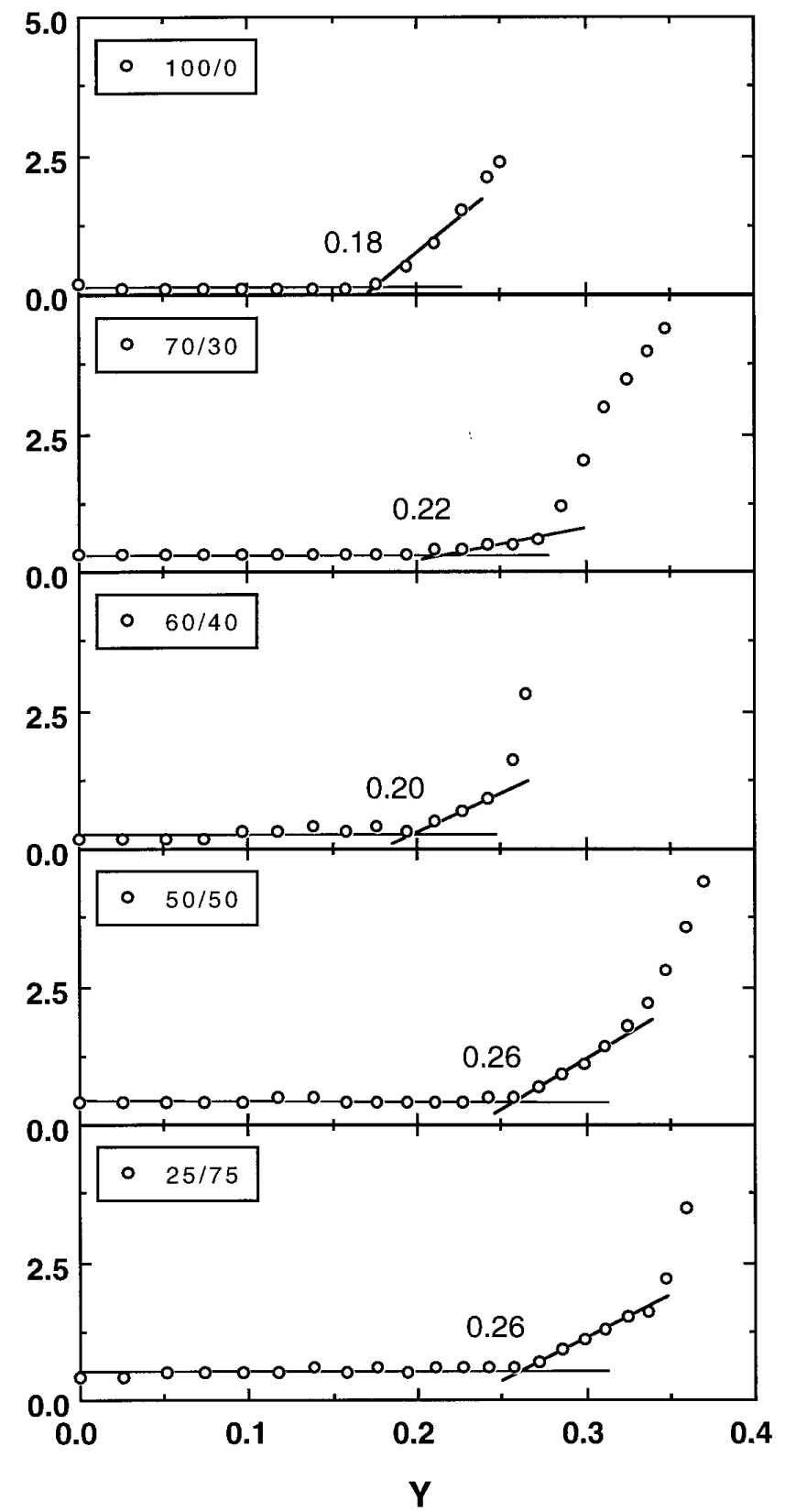

FIG. 6. Turbidimetric titrations for $0.50 \mathrm{~g} / \mathrm{L}$ PAMPS-co-AAm $+20 \mathrm{mM}$ $\mathrm{C}_{12} \mathrm{E}_{8}$ with $40 \mathrm{mM}$ CTAC in $0.20 \mathrm{M} \mathrm{NaCl}$. Copolymer compositions and $Y_{\mathrm{c}}$ are shown in inserts.

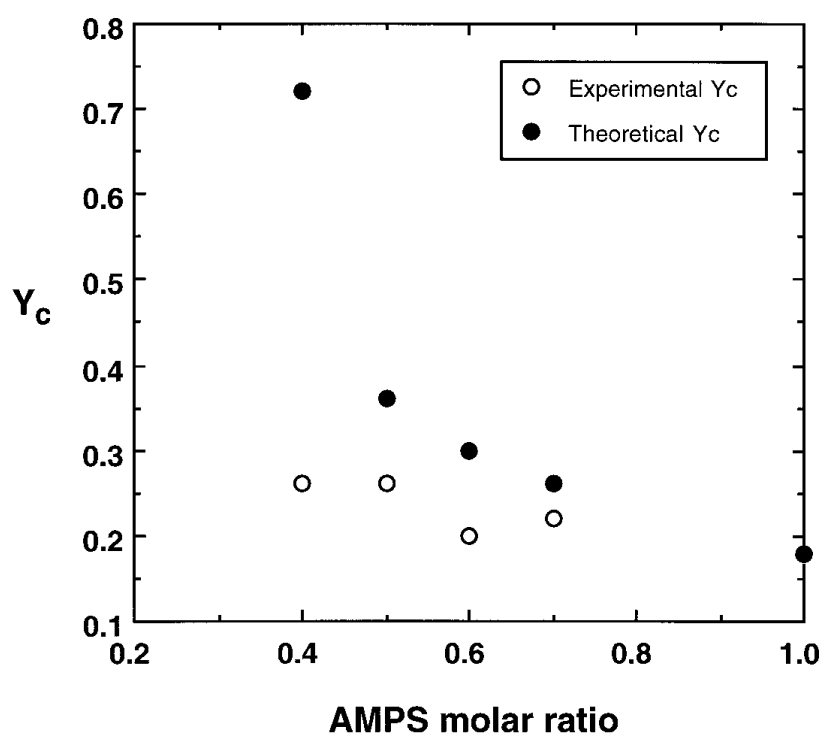

FIG. 7. The dependence of $Y_{\mathrm{c}}$ on (\% AMPS $)^{-1}$ from Fig. 6. Broken line is extrapolation from result for PAMPS based on Eq. (1).

of AMPS is small. However, since $Y_{\mathrm{c}}$ corresponds to the onset of micelle binding, it may be strongly influenced by the presence of a relatively small concentration of such runs. Future comparisons between low- and high-conversion copolymers for which sequence distributions have been obtained by NMR may give additional insight into the effects of compositional variability.

\section{CONCLUSIONS}

The binding of polyelectrolytes to homogeneously oppositely charged surfaces generally becomes stronger with increasing polymer linear charge density. Polyacrylic acid, and, by extension, other polycarboxylic acids, may present an anomalous case in that their effective charge densities are strongly modulated by counterion binding. This effect is not to be confused with "counterion condensation," for which only the charge density of the polymer and counterion valence are important; the present phenomenon appears to be highly specific to the interaction of polymeric carboxylate and metal ions. While this specific ion binding reduces the effective charge density, the mobility of bound protons in partially ionized polyacrylic acid can enhance the effective charge density through a polarization effect in which the local degree of ionization of the polyion increases in the vicinity of a positively charged surface. Somewhat similarly, a charged surface may preferentially adsorb those sequences of a copolymer that are rich in the oppositely charged monomer.

\section{ACK NOWLE DGMENTS}

This work was supported by grant DMR-9619722 from the National Science Foundation, and by a Grant-in-Aid from Shiseido Corp. The assistance of T. Sato and Y. Morishima is gratefully acknowledged. 


\section{REFERENCES}

1. Wiegel, F. W., J. Phys. A: Math. Gen. 10, 299 (1977).

2. Muthukumar, M., J. Chem. Phys. 86, 7230 (1987).

3. Evers, O. A., Fleer, G. J., Scheutjens, J. M. H. M., and Lyklema, J., J. Colloid Interface Sci. 111, 446 (1986).

4. Odijk, T., Langmuir 7, 1 (1991).

5. von Goeler, F., and Muthukumar, M., J. Chem. Phys. 100, 7796 (1994).

6. Muthukumar, M., J. Chem. Phys. 103, 4723 (1995).

7. Dubin, P. L., Chew, C. H., and Gan, L. M., J. Colloid Interface Sci. 128, 566 (1989).

8. Dubin, P. L., Curran, M. E., and Hua, J., Langmuir 6, 707 (1990).

9. Dubin, P. L., Vea, M. E. Y., Fallon, M. A., Thé, S. S., Rigsbee, D. R., and Gan, L. M., Langmuir 6, 1422 (1990).

10. McQuigg, D. W., Kaplan, J. I., and Dubin, P. L., J. Phys. Chem. 96, 1973 (1992).

11. Mattison, K. W., Brittain, I. J., and Dubin, P. L., Biotechnol. Prog. 11, 632 (1995).

12. Park, J. M., Muhoberac, B. B., Dubin, P. L., and Xia, J., Macromolecules 25, 290 (1992).

13. Dubin, P. L., Chew, C. H., and Gan, L. M., J. Colloid Interface Sci. 128, 566 (1989).

14. Dubin, P. L., Rigsbee, D. R., and McQuigg, D. W., J. Colloid Interface Sci. 105, 509 (1985).

15. Zhang, H., Bahadur, P., Parotte, J., and Dubin, P. L. in preparation.

16. Mattison, K. W., Dubin, P. L., and Brittain, I. J., J. Phys. Chem., in press.

17. Xia, J., Zhang, H., Rigsbee, D. R., Dubin, P. L., and Shaikh, T., Macromolecules 26, 2759 (1993). and references therein; Dubin, P. L., Vea, M. E. Y., Fallon, M. A., Thé, S. S., Rigsbee, D. R., and Gan, L. M., Langmuir 6, 1422 (1990).

18. Bungenberg de Jong, H. L., in "Colloid Science" (H. H. Kruyt, Ed.), Vol. 2. Elsevier, Amsterdam, 1952.

19. Morawetz, H., and Hughes, Jr. W. L., J. Phys. Chem. 56, 64 (1952).

20. Clark, K. C., and Glatz, C. E., Biotechnol. Prog. 3, 241 (1987).

21. Sternberg, M., and Hershberger, D., Biochim. Biophys. Acta 342, 195 (1974).

22. Kabanov, V. A., Mustafaev, M. I., Belova, V. V., and Evdakov, V. P., Mol. Biol. (Moscow) 12, 1264 (1978).

23. Pautov, V. D., Kuznetsova, N. P., Mishayeva, R. N., and Anufriyeva, Ye. V., Vysokol. Soyed. A25, 1599 (1983).

24. Blaakmeer, J., Böhmer, M. R., Cohen Stuart, M. A., and Fleer, G. J., Macromolecules 23, 2301 (1990).

25. Hayakawa, K., and Kwak, J. C. T. in "Cationic Surfactants. Physical Chemistry" (D. N. Rubingh and P. M. Holland, Eds.). Chap. 5. M. Dekker, New York (1991).

26. Chu, D.-Y., and Thonas, J. K., J. Am. Chem. Soc. 108, 6270 (1986).

27. Almgren, M., Hansson, P., Mukhtar, E., and van Stam, J., Langmuir 8, 2405 (1992).

28. Fundin, J., Hansson, P., Brown, W., and Lidegran, I., Macromolecules 30, 1118 (1997).

29. Thalberg, K., van Stam, J., Lindblad, C., Almgren, M., and Lindman, B., J. Phys. Chem. 95, 68975 (1991).

30. Böhmer, M. R., Evers, O. A., and Scheutjens, J. M. H. M., Macromolecules 23, 2288 (1990). (b) Evers, O. A., Fleer, G. J., Schutjens, J. M. H. M., and Lyklema, J., J. Colloid Interface Sci. 111, 446 (1986).
31. Foissy, A., El Attar, E., and Lamarche, J., J. Colloid Interface Sci. 96, 275 (1983).

32. de Laat, A. W. M., van den Heuvel, G. L. T., and Böhmer, M. R., Colloids Surf. A 98, 61 (1995).

33. Shatayeva, L. K., Radzyavishyus, K. I., and Samsonov, G. V., Vysokomol. Soed. A27, 702 (1985).

34. Li, Y., and Dubin, P. L., in "Rheology of Surfactant Solutions," (C. A. Herb, and R. K. Prud'homme, Eds.). American Chemical Society, Washington, D.C. (1994).

35. Zhang, H., Dubin, P. L., and Ohbu, K., in preparation.

36. Li, Y., Dubin, P. L., Spindler, R., and Tomalia, D. A., Macromolecules 28, 8426 (1995).

37. Zhang, H., Dubin, P. L., Kaplan, J., Moorefield, C. N., and Newkome, G. R., J. Phys. Chem. 101, 3494 (1997).

38. Gregor, H. P., in "Polyelectrolytes," (E. Selegny, Ed.). p. 87. D. Reidel, Dordrecht (1974)

39. (a) Strauss, U. P., and Leung, Y. P., J. Am. Chem. Soc. 87, 1476 (1965). (b) Rinaudo, M., and Milas, M., J. Chim. Phys. 66, 1489 (1969). (c) Begala, A. J., and Strauss, U. P., J. Phys. Chem. 76, 254 (1972). (d) Eldridge, R. J., and Treloar, F. E., J. Phys. Chem. 80, 1513 (1976). (e) Strauss, U. P., and Schlesinger, M. S., J. Phys. Chem. 82, 571 (1978).

40. Manning, G. S. private communication.

41. Sato, T., Mattison, K. W., Dubin, P. L., Kamachi, M., and Morishima Y., submitted.

42. Anufrieva, E. V., Birshtein, T. M., Nekrasova, T. N., Ptitsyn, O. B., and Sheveleva, T. V., J. Polym. Sci. C 16, 3519 (1968).

43. Kono, N., and Ikegami, A., Biopolymers 4, 823 (1966).

44. Dubin, P. L., and Davis, D. D., Macromolecules 17, 1294 (1984).

45. Yoshida, K., Dubin, P. L., Morishima, Y., and Mizusaki, M., Macromolecules 30, 6208 (1997).

46. Manning, G. S., Acc. Chem. Res. 12, 443 (1979).

47. Yoshida, K., and Dubin, P. L., Colloids Surf., in press.

48. McQuigg, D., Thesis, Purdue University, 1991.

49. Gregor, H. P., and Frederick, M., J. Polym. Sci. 23, 451 (1957).

50. Kagawa, I., and Gregor, H. P., J. Polym. Sci. 23, 477 (1957).

51. Kiefer, J. J., Somasundaran, P., and Ananthapadmanabhan, K. P., Langmuir 9, 1187 (1993).

52. Remy, H., in "Treatise on Inorganic Chemistry," Vol. I, p. 75. Elsevier, New York (1956).

53. Dubin, P. L., Thé, S. S., McQuigg, D. W., Chew, C. H., and Gan, L. M., Langmuir 5, 89 (1989).

54. While alkyl ammonium ions with long-chain alkyl groups may be solubilized in micelles, there is no effect of $\mathrm{TMA}^{+}\left(\mathrm{vs} \mathrm{Na}^{+}\right)$on the cloud point of Triton X100 (Schott, H., Royce, A. E., and Han, S. K., J. Colloid Interface Sci. 98, 196 1984) and little effect of tetraethylammonium chloride (vs $\mathrm{NaCl}$ ) on the cloud point of $\mathrm{C}_{12} \mathrm{E}_{5}$ (Aveyard, R., and Lawless, T. H., J. Chem. Soc. Faraday Trans 1 82, 2951 1986); these results indicate that $\mathrm{TMA}^{+}$is not partitioned into nonionic surfactants.

55. Zhang, H., Dubin, P. L., Manning, G. S., Newkome, G. R., and Moorefield, C. N., in preparation.

56. J. Joanny, private communication.

57. Miura, N., and Dubin, P., unpublished.

58. Morishima, Y., and Sato, T., in preparation. 